\title{
Magnetic resonance imaging technology in transtibial socket research: A pilot study
}

\author{
Arjan W. P. Buis, PhD, CPO; ${ }^{*}$ Barrie Condon, PhD $;^{2}$ Dave Brennan, PhD; ${ }^{2}$ Brendan McHugh, PhD; ${ }^{1}$ \\ Donald Hadley, PhD, FRCR ${ }^{3}$ \\ ${ }^{1}$ National Centre for Training and Education in Prosthetics and Orthotics, University of Strathclyde, Glasgow, \\ Scotland; ${ }^{2}$ Clinical Physics Department and ${ }^{3}$ Neuroradiology Department, Institute of Neurological Sciences, \\ Southern General Hospital, Glasgow, Scotland
}

\begin{abstract}
Investigations into the shape and volume of transtibial prosthetic sockets are complicated because of the difficulty in establishing an accurate reference grid. Magnetic resonance imaging (MRI) presents a possible solution to this problem. However, the reliability of MRI in defining the residual-limb/cast interface depends on the scanned image not being distorted by the materials present. We investigated the potential of MRI technology to establish the desired reference grid. Distortion from the so-called "chemical shift" may influence the MRI when certain materials are used during the casting process. These materials include plaster of paris (POP) and silicone (in the form of an interface liner). POP is commonly used to capture the shape of the residual limb. However, if the casting technique requires the use of a silicone liner, the liner is placed over the residual limb first and then the POP is applied over the liner. Experimental results indicate that the materials used do not distort or interfere with the scanned image. The object segmentation process that extracts the bone and skin from an MRI scan and enables the establishment of the required reference grid was explored. Results show that extracting the bone structure and using it as the reference grid to quantify the differences in volume and shape of the soft tissues of the residual limb is feasible.
\end{abstract}

Key words: chemical shift, magnetic resonance imaging, object segmentation, plaster of paris, reference grid, residual limb, residual-limb/cast interface, silicone, transtibial prostheses, transtibial prosthetic sockets.

\section{INTRODUCTION}

The first theoretical model of transtibial socket shape, in relation to biomechanics and residual-limb characteristics, was developed in the 1960s by Radcliffe and Foort [1-2]. Three decades later it was refined by Klasson [3]. Klasson advocated a shape-optimization process for achieving an ideal pressure distribution and effective coupling between the residual limb and socket during normal use. Limited evidence-based research validates these theories. A prerequisite of validating these theories is an understanding of the consistency of the socket-manufacturing process. Casting consistency and subsequent socket shape, including the influences of manual dexterity during rectification, have been reported by Buis et al. [4] and Convery et al. [5]. These studies

\footnotetext{
Abbreviations: $3-\mathrm{D}=3$-dimensional, $\mathrm{A}-\mathrm{P}=$ anterior-posterior, $\mathrm{CAD}=$ computer-aided design, DICOM = Digital Imaging and Communications in Medicine, MRI = magnetic resonance imaging, NCTEPO $=$ National Centre for Training and Education in Prosthetics and Orthotics, $\mathrm{POP}=$ plaster of paris, PTB = patellar-tendon-bearing.

${ }^{*}$ Address all correspondence to Dr. Arjan W. P. Buis, National Centre for Training and Education in Prosthetics and Orthotics, University of Strathclyde, Glasgow, Scotland G4 0LS; +44-141-548-3693; fax: +44-141-552-1283.

Email: arjan.buis@strath.ac.uk

DOI: 10.1682/JRRD.2005.08.0145
} 
describe experiments executed in a controlled environment with a purpose-designed residual-limb model for studying casting consistency and multiple identical copies for studying rectification. However, the consistency of the socket manufacturing process has not previously been quantified.

Investigations into the shape and volume of transtibial prosthetic sockets are notoriously complicated because of the difficulty in establishing an accurate reference grid for inter- and intrasocket comparisons and evidence-based outcome measurements. The use of magnetic resonance imaging (MRI) presents a possible solution to this problem. However, the reliability of MRI in defining the residuallimb/cast interface depends on the scanned image not being distorted by the materials present. Another issue that should be mentioned is that MRI technology is somewhat expensive.

An MRI scan is a radiology technique that uses magnetism, radio waves, and computer hardware and software to produce detailed images of the body structure. MRI is a nondestructive technique with the advantage of avoiding ionizing radiation exposure (X-rays). Therefore, serial studies become possible. The key principle of MRI technology is the specific behavior of hydrogen nuclei influenced by very strong magnetic fields $(\sim 30,000$ times as strong as the Earth's field). Hydrogen nuclei have an electric charge and "spin" and the spinning charges produce a magnetic field. As a direct result, the hydrogen nuclei act like tiny magnets. The human body is $\sim 80$ percent water and, therefore, has an extremely large number of hydrogen nuclei. When a human body is exposed to the highstrength magnetic field produced by the scanner, the hydrogen nuclei either align with the field (low-energy state) or align in the opposite direction (high-energy state). An oscillating magnetic field at the correct frequency can make nuclei change from low- to high-energy states. The energy given to the system by the oscillating magnetic field will be returned as the nuclei relax back into the low-energy state and can be detected by the receiver unit of the MRI scanner. The amount of energy returned is an index of how many hydrogen nuclei are present. The rate at which the energy is returned is related to two relaxation processes with two time constants: spinlattice relaxation time and spin-spin relaxation time. The detected changes in the hydrogen nuclei are then processed by a computer and an image is produced.

This image can be represented either as a 2-dimensional slice similar to a radiograph for diagnostic purposes or as a volume-rendered 3-dimensional (3-D) graphical image. This data set is a 3-D array of so-called "voxels." A voxel may be considered a 3-D or volume pixel. The selection of a specific voxel dimension depends on the size and volume of the planned body segment to be scanned, the time it takes to scan, and the required detail (resolution).

MRI studies where the dimensional accuracy was important have been reported by a number of authors [610]. Accuracy depends on numerous factors, including scanning parameters and segment size. Cohen et al. investigated the accuracy of MRI technology in a study of articular surface topography and cartilage thickness in six human cadaveric knee joints compared with the calibrated stereophotogrammetric method (voxel dimensions $0.47 \times 0.47 \times 1.0 \mathrm{~mm}$ ) [11]. They reported that the average accuracies of cartilage and subchondral bone surface measurements were 0.22 and $0.14 \mathrm{~mm}$, respectively, and the thickness measurements demonstrated an average accuracy of $0.31 \mathrm{~mm}$. They also found that while most of the error may be attributed to random measurement error, the accuracy was somewhat affected by systematic errors. This study contributes to the confidence that clinical MRI can provide sufficiently accurate measurements.

The most common influences on the accuracy and validity of a scanned image are the so-called "chemical and mechanical shift.” Mechanical shift is caused by a physical disturbance, such as the movement of the segment of interest during the scanning procedure. The result can be compared with a blurred photograph of a moving object as a result of slow shutter speed or camera movement. Chemical shift is a little more complicated; the biological composition of tissue matter will influence the return signal to the MRI receiver unit when the tissue is exposed to a pulsed high-strength magnetic field. Specifically, tissues with a high fat content will return a strong signal with a shifted resonant frequency compared with other tissue. Since frequency is used to encode position along one of the spatial axes, this results in an artifactual spatial shift for fat. However, chemical shift is a systematic phenomenon and could in principle be compensated for.

In examining the feasibility of using MRI technology for prosthetic socket comparison, two questions had to be considered:

- Can a reliable image be obtained when common casting materials are used, including silicone liners?

- Is establishing the required reference grid for socket shape and volume studies feasible? 


\section{METHODS}

The system we used was a GE Medical Systems Signa CVi/NVi 1.5T (General Electric, Co; Fairfield, Connecticut), which had a 3-D inversion recovery fast spoiled gradient pulse sequence, with a repetition time of $11.9 \mathrm{~ms}$, an echo time of $2.5 \mathrm{~ms}$, a preparation time of $400 \mathrm{~ms}$, a flip angle of $20^{\circ}$, a $160 \mathrm{~mm}$ field of view with $256 \times 256$ voxel resolution, a slice thickness of $2 \mathrm{~mm}$, and a 1 signal average. Acquisition time was 9 min and $52 \mathrm{~s}$. The slices were aligned with an initial 3-plane localizer. Images were acquired with a flexible phased-array surface coil wrapped around the limb.

When a body segment is scanned, the MRI equipment captures images in the form of slices depending on the machine settings and the detail required. This single slice information is filed in Digital Imaging and Communications in Medicine (DICOM) format. A scanning sequence of a body segment consists of a number of individual slices that together are called an image volume. The display software of the imager is limited in its capabilities. Therefore, all DICOM data were exported to the state-of-the-art visualization software Analyze ${ }^{\circledR} 5.0$ (AnalyzeDirect, Inc; Overland Park, Kansas) developed at the Mayo Clinic Biomedical Imaging Resource in Rochester, Minnesota. This software was used for multidimensional display, processing, and measurement of the recorded data.

\section{Materials Influencing Scanned MRI}

The possibility exists that chemical shift will influence the MRI when common casting materials, including silicone and plaster of paris (POP), are used. The first experiment included an independent reference grid called a "phantom" and a number of square pieces of different silicone liner materials. These are illustrated in Figure 1, where a cross-sectional slice of the reference grid is shown. The round feature is the phantom, and the silicone is indicated by the white arrow. The purpose of this experiment was to investigate (1) whether silicone was visible on the MRI and (2) whether significant artifactual spatial chemical shift was present within the test specimen in a controlled environment. The latter was anticipated because the hardness or shore of silicone is influenced by silicone oil (fat) introduced during the manufacturing process. A

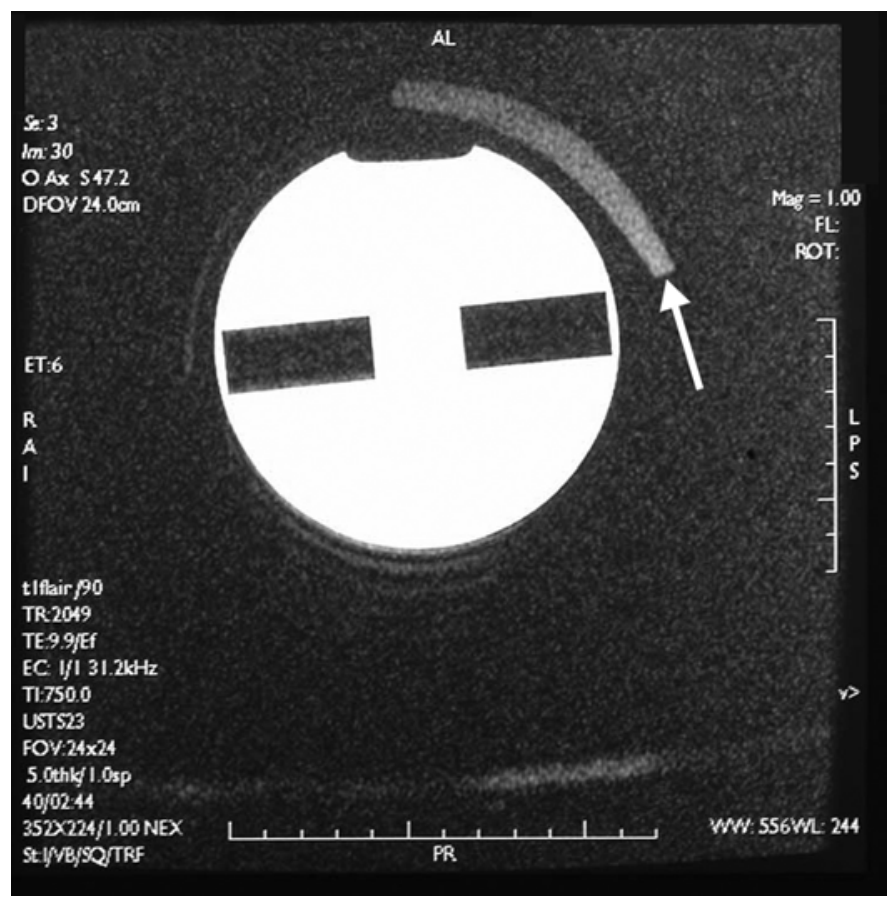

Figure 1.

Cross-sectional slice of independent reference grid used to assess effect of chemical shift on magnetic resonance images. Round feature is phantom, and silicone is indicated by white arrow.

piece of liner material was placed on the phantom and a scan was taken.

The second experiment involved a normal transtibial amputee and a fresh POP cast for investigating the effects of POP on the scanned image. Using the method taught and practiced at the National Centre for Training and Education in Prosthetics and Orthotics (NCTEPO) at the University of Strathclyde, we hand-casted the subject with the aim of producing a patellar-tendon-bearing (PTB) type of prosthetic socket. This method is described in detail in the Transtibial Prosthetics manual distributed by the NCTEPO [12]. As soon as the cast had cured, the subject was placed in the prone position on the movable table of the MRI machine. The subject's residual limb, including cast, was placed in a clear acrylic tube $(15 \mathrm{~cm}$ diameter) with a special patellar support designed to avoid soft tissue deformation from resting contact. The extension moment of the knee joint in the prone position might contribute to reduced positional differences when multiple scans are required. A flexible signal receiver coil, normally used for spinal investigations, was wrapped around the acrylic tube. The close proximity of this coil to the body segment greatly increased the 
detected signal compared with the internal machine receiver coil only. Another important feature of placing the coil around the tube was avoiding contact pressure and subsequent soft tissue deformation. After positioning, we acquired a scan. Each subject provided written informed consent according to the guidelines of the South Glasgow University Hospitals National Health Service Trust Ethics Committee, Glasgow, Scotland, which granted full ethical approval for the study (EC/02/S/109).

Experimental results indicated that the stronger the return signal from the fresh POP bandage, the clearer the boundary would be between the cast and the skin. To explore ways to enhance the return signal, we conducted the following experiment. Nine small plastic specimen containers were filled with stacked disks cut from uncured POP bandage to a height of $3 \mathrm{~cm}$. Each container was filled with water doped with copper sulfate of varying concentrations ranging from $0.25 \mathrm{~g} / \mathrm{L}$ to $2.0 \mathrm{~g} / \mathrm{L}$. The liquid was drained and the saturated POP bandage was left to cure for $10 \mathrm{~min}$. After curing the bandage, we placed the nine containers and an additional block of animal fat (lard) as a reference in the MRI machine and scanned them.

\section{Mechanical Shift}

Preconditions for comparing and evaluating variations in socket shape and volume are first, that a fixed reference grid exists and second, that the image is not influenced by mechanical shift. Logically, the bone structure of the residual limb can potentially meet the criteria for a reference grid because it is the only structure that is not influenced by deformations during the various casting processes.

Mechanical shift can be subdivided in two categories; the first category applies to all MRIs and can be expressed as movement or disturbance of the segment of interest during scanning. This movement is often due to muscle contractions. The second can be described as positional mechanical shift, which occurs when a number of scans of the same segment are superimposed or aligned for comparison or follow-up studies, such as inter- and intraconceptual prosthetic socket comparisons.

We performed preliminary experiments to investigate the stability or movement of the tibia inside a POP cast prior to MRI scanning. This movement is important because it determines a possible significant and critical error margin for comparison studies. However, we must stress that this movement is not the same as that which would occur during stance or gait. A silicone liner with a square cutout window at the end of the tibia was placed over the residual limb of an amputee subject. The end of the tibia was selected because most of the movement was likely to occur in this region. The cutout was plugged with a piece of foam, as illustrated in Figure 2, and a POP cast was applied. After curing the cast, we removed the foam plug, exposing the distal end. A displacement gauge was fixed to the POP cast where the probe of the measurement gauge was touching the skin overlying the most distal part of the tibial crest (Figure 3). The subject was placed in a prone position with his residual limb resting on the special patellar support as described earlier and asked to relax his muscles. We asked the subject to sit up and then to return to the prone position. We repeated this procedure several times to investigate positional repeatability in the anterior-posterior (A-P) direction, which is considered the direction of greatest tissue deformation.

\section{Establishment of Required Reference Grid}

As for all comparisons, a baseline or reference must be set and the most logical anatomical structure for such a reference in transtibial socket research is the tibia, which is the only stable entity in the transtibial residual limb. The Analyze ${ }^{\circledR}$ software can segment or highlight specific structures, such as the tibia, from an image volume.

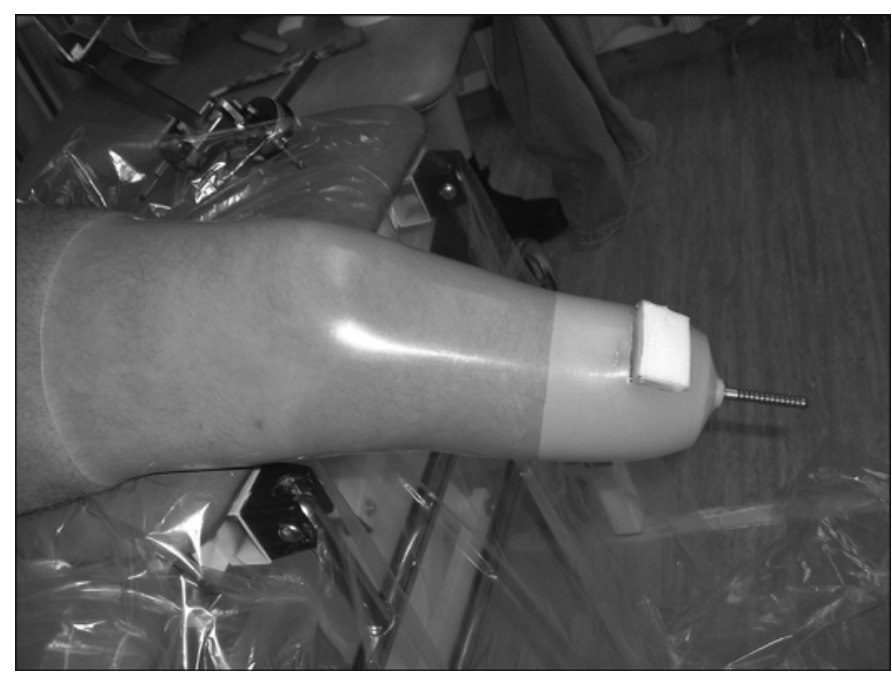

Figure 2.

Silicone liner with square cutout window at end of tibia was placed over residual limb of amputee subject. Cutout was plugged with piece of foam, and plaster of paris cast was applied. 


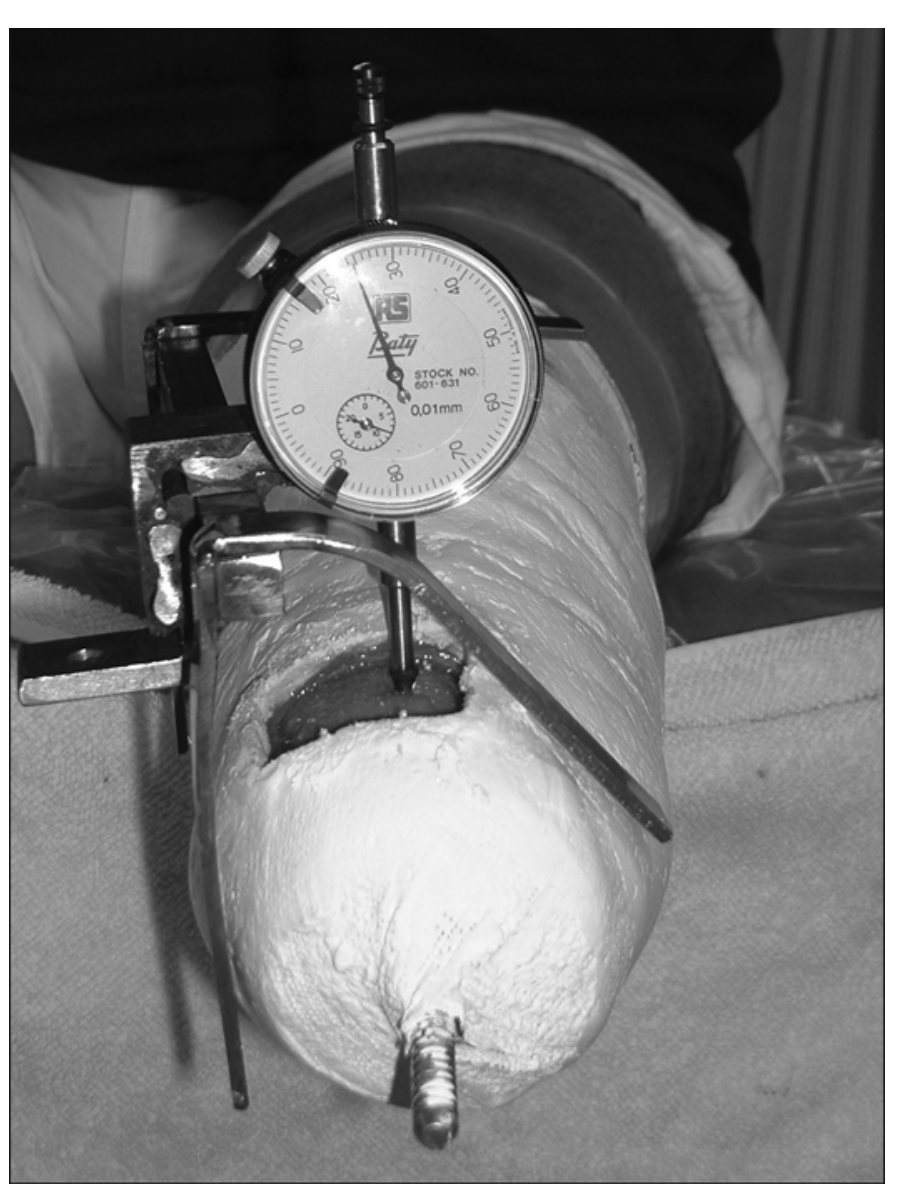

Figure 3.

Displacement gauge was fixed to plaster of paris cast where probe of measurement gauge was touching skin overlying most distal part of tibial crest.

Multiple image volumes can be registered in 3-D, which allows structural data to be computationally aligned to exactly match the base volume. This base volume is referred to as the selected reference grid. As a result, the possibility exists to relate different wrap casts of the subject to underlying anatomical coordinates for the first time. Hence, quantifying shape and volume differences should be feasible.

To determine the base volume, we performed the following experiment. We hand-casted and scanned an amputee subject as described previously. Multiple scans with the same subject and same POP cast were recorded. The subject was asked to sit up from the prone scanning position after each recording session. We exported the data to the Analyze ${ }^{\circledR}$ software to investigate the repeatability of all the steps in the process, including the accuracy of the registration process and the mechanical positional shift differences between scanned image volumes.

The Analyze ${ }^{\circledR}$ software is capable of loading two image volumes at the same time (Figure 4(a)-(c)). Figure 4(a) is the base image volume and illustrates a longitudinal slice section of the residual limb. Figure 4(c) is the data from a second image volume of the same slice, and Figure 4(b) visualizes the combined image volumes of Figure 4 (a) and (c). The quality of the registration was viewed with the cursor links tool (crosshairs).

\section{RESULTS}

One concern was that commonly used casting and liner materials might influence the scanned image. The first experiments investigated whether silicone was visible and artifactual shifts were noted. The results indicated that the casting materials (silicone and POP) did not distort or interfere with the scanned image. No detectable mechanical shift as a result of movement during the scanning process was demonstrated. Preliminary experiments on the stability or movement of the tibia in the A-P direction inside a plaster cast with the use of a displacement gauge showed a maximum deviation of $0.3 \mathrm{~mm}$. No detectable chemical shift was identified after expert examination of the scanned images. The signal contrast of POP was enhanced by 400 percent by adding copper sulfate at a concentration of $2 \mathrm{~g} / \mathrm{L}$ to the casting water.

Researchers may be able to extract the bone structure, which is a solids volume procedure, and use it as a reference grid for 3-D alignment of multiple image volumes. See Figure 4(a)-(c) for an example of an image volume before registration and Figure 4(d)-(f) for an example after registration. The combined image volumes before registration (Figure 4(b)) show a blurred image. This blurring is the result of misalignment of the two image volumes. Figure 4(e) shows the result after image volume registration. The maximum variation found after two consecutive scans of the same POP cast was on the order of $1.2 \mathrm{~mm}$ (2 pixels). However, 95 percent of the scan showed less than $0.62 \mathrm{~mm}$ (1 pixel) variation.

\section{DISCUSSION}

This study explored the potential of MRI technology for establishing a reference grid for prosthetic socket 
(a)

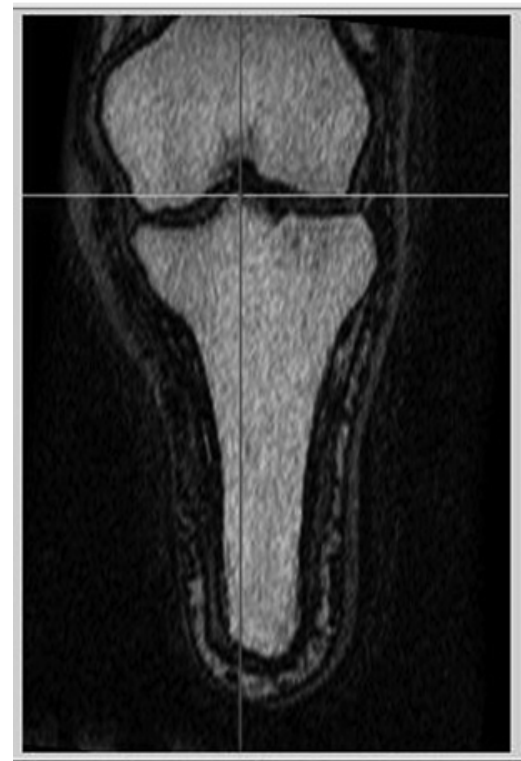

(d)

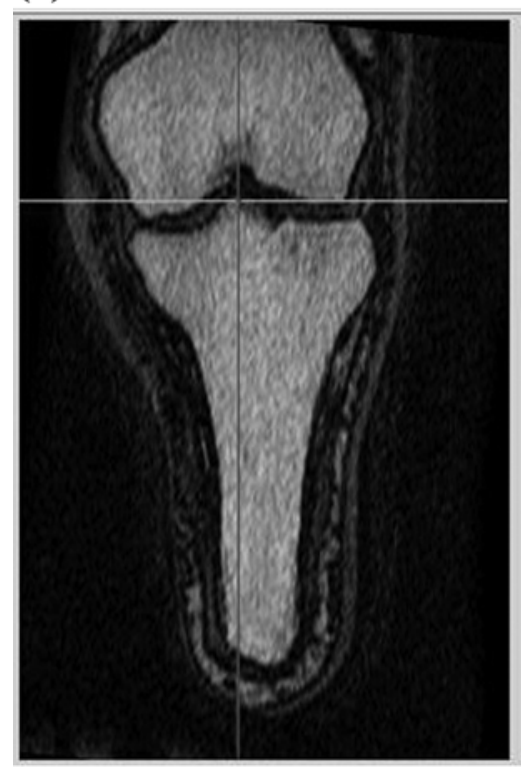

(b)

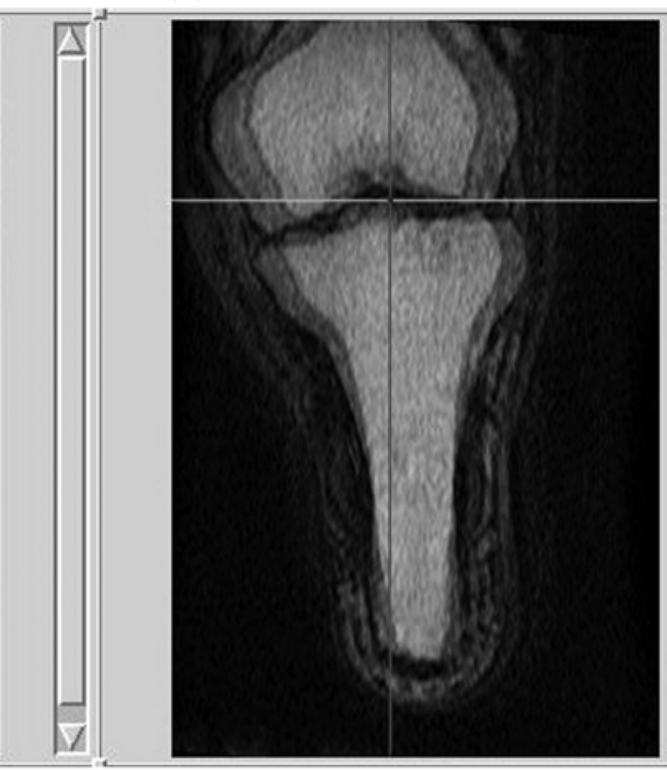

(e)

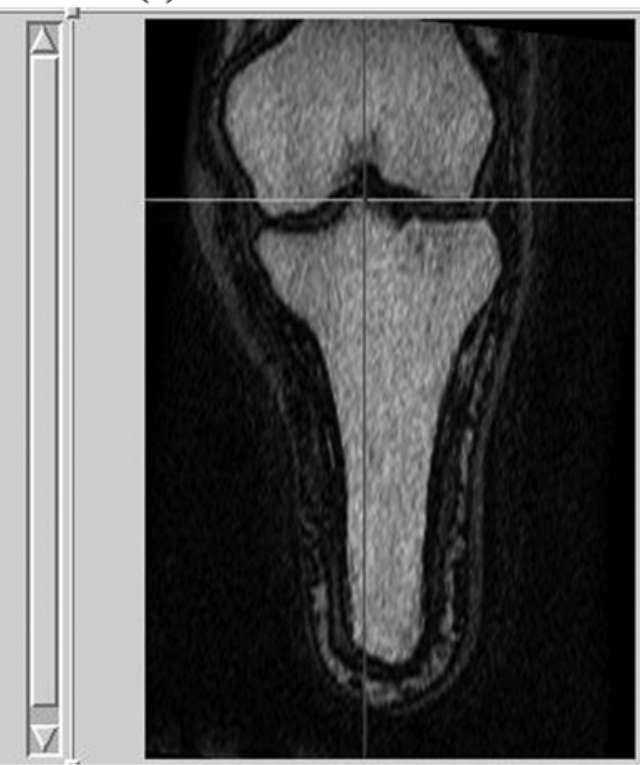

(c)

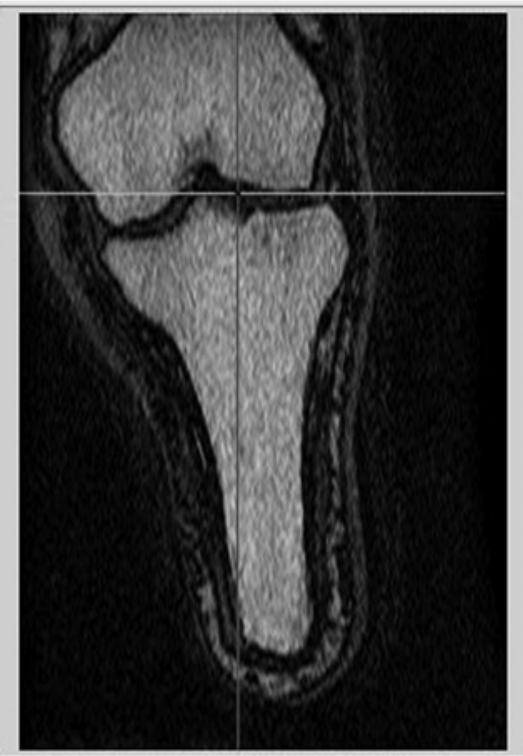

(f)

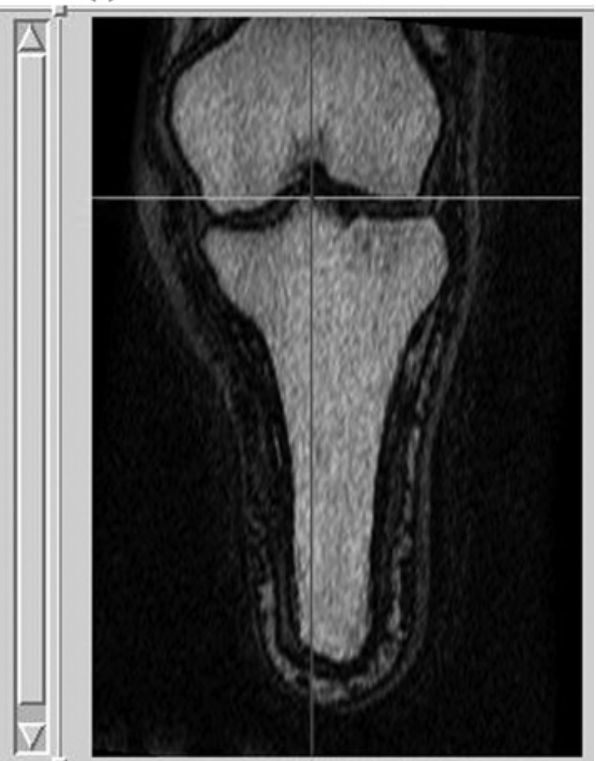

Figure 4.

(a) Base image volume of longitudinal slice section of residual limb. (b) Combined image volumes before registration of (a) and (c) (second image volume of same slice). (d) and (f) Image volumes of longitudinal slice sections of residual limb. (e) Combined image volumes of (d) and (f) after registration.

research. Two major questions concerning MRI technology were explored: What are the influences on the scanned image when common casting materials are used, and what is the feasibility of establishing the required reference grid for socket shape and volume studies?

This study demonstrated that quantifying differences in casting shape with the tibia as a reference grid is feasi- ble. Movement of the tibia in a final socket during gait has been reported by numerous authors [13-16]. However, we must recognize that this skeletal movement is quite different from the movement inside a freshly applied transtibial POP cast, i.e., a cast that is cured but not yet removed. The results of the bone stability experiment demonstrated that the bone-to-cast movement in the 
A-P direction was not significant for this study, because the minimum in-plane pixel/voxel size of the MRI was on the order of $0.65 \mathrm{~mm}$ compared with the maximum measured movement of $0.30 \mathrm{~mm}$. We could argue that the longitudinal displacement was most likely minimal because (1) the cast in relation to the residual limb/bone was locked over the condyles and (2) the cast was not removed. To a certain extent, this argument can also be applied to rotational stability. The thumb and finger positions executed and inherent to the PTB casting concept will prevent, in combination with the condyle indentation, the cast from rotating. However, longitudinal movements and transverse plane rotations should be investigated in future work. The enhancement of the output signal of POP (400\%) contributed greatly to the definition of the boundary between skin and POP, lifting segmentation out of the ambiguous gray area of selecting the right structure.

This study also attempted to find a practical balance between the best scanning parameters and a scanning time that was realistically tolerable to subjects. Unfortunately, we do not live in a perfect world, so compromises have to be made. The most realistic maximum scanning duration was $10 \mathrm{~min}$. A $0.6 \mathrm{~mm}$ in-plane resolution and a slice thickness of $2 \mathrm{~mm}$ gave the best detail possible for a 10-minute session. The in-plane resolution was chosen because previous studies by Buis et al. [4] and Convery et al. [5] show that maximum in-plane inaccuracies during casting and rectification are on the order of $5 \mathrm{~mm}$. Probably more important, the longitudinal rectification differences were found to be on the order of $7 \mathrm{~mm}$; differences this small are inherently difficult for the prosthesis user to identify. As a result, the $2 \mathrm{~mm}$-slice thickness was appropriate for this study.

\section{CONCLUSIONS AND SUGGESTIONS}

The results of the described experiments as well as the accuracy studies described by other authors support the considerable value of MRI technology for investigation of the mechanisms contributing to a "good" prosthetic socket fit. Although MRI has benefits, it also clearly has limitations, including costs and realistic and practical scanning duration. Suggestions for future studies are numerous and might include the exploration of a variety of materials to enhance the contrast between skin and casting material. Another option might be an in-depth val- idation study to confirm the previously described establishment of the reference grid. Also, polyurethane casting socks could be included since they are often used or proposed for computer-aided design (CAD)/computer-aided manufacturing systems that allow scanning of the outer surface of the cast and subsequent software correction. In particular, the combination of CAD (surface scan) and MRI (internal structures) could benefit this kind of research.

\section{ACKNOWLEDGMENTS}

This material is the result of work supported with resources and the use of facilities at the NCTEPO, University of Strathclyde, Glasgow, Scotland.

The authors have declared that no competing interests exist.

\section{REFERENCES}

1. Radcliffe CW. The biomechanics of below-knee prostheses in normal, level, bipedal walking. Artif Limbs. 1961;6(2): 16-24. [PMID: 13972953]

2. Radcliffe CW, Foort J. The patellar-tendon-bearing belowknee prosthesis. Berkeley (CA): University of California, Biomechanics Laboratory; 1961.

3. Klasson B. Appreciation of prosthetic socket fitting from basic engineering principles. Glasgow (Scotland): University of Strathclyde; 1995.

4. Buis AW, Blair A, Convery P, Sockalingam S, McHugh B. Pilot study: Data-capturing consistency of two trans-tibial casting concepts, using a manikin stump model: A comparison between the hands-on PTB and hands-off ICECAST compact concepts. Prosthet Orthot Int. 2003;27(2):100-106. [PMID: 14571939]

5. Convery P, Buis AW, Wilkie R, Sockalingam S, Blair A, McHugh B. Measurement of the consistency of patellartendon-bearing cast rectification. Prosthet Orthot Int. 2003; 27(3):207-13. [PMID: 14727701]

6. Kishida Y, Nishii T, Sugano N, Nakanishi K, Sakai T, Miki $\mathrm{H}$, Ochi T, Yoshikawa H. Measurement of lesion area and volume by three-dimensional spoiled gradient echo MR imaging in osteonecrosis of the femoral head. J Orthop Res. 2003;21(5):850-58. [PMID: 12919873]

7. Cyteval C, Thomas E, Picot MC, Derieffy P, Blotman F, Taourel P. Normal vertebral body dimensions: A new measurement method using MRI. Osteoporos Int. 2002; 13(6):468-73. [PMID: 12107660] 
8. Muensterer OJ, Eckstein E, Hahn D, Putz R. Computeraided dimensional assessment of knee-joint cartilage with magnetic resonance imaging. Clin Biomech (Bristol, Avon). 1996;11(5):260-66. [PMID: 11415630]

9. Eckstein F, Schnier M, Haubner M, Priebsch J, Glaser C, Englemeier KH, Reiser M. Accuracy of cartilage volume and thickness measurements with magnetic resonance imaging. Clin Orthop. 1998;(352):137-48. [PMID: 9678042]

10. Lee VSP, Solomonidis SE, Spence WD, Paul JP. Mechanical behavior of amputee stump/socket using finite element method [abstract]. J Biomech. 1994;27(6):838.

11. Cohen ZA, McCarthy DM, Kwak SD, Legrand P, Fogarasi F, Ciaccio EJ, Ateshian GA. Knee cartilage topography, thickness, and contact areas from MRI: In-vitro calibration and in-vitro measurements. Osteoarthritis Cartilage. 1999; 7(1):95-109. [PMID: 10367018]

12. National Centre for Training and Education in Prosthetics and Orthotics (NCTEPO). Transtibial prosthetics manual. Glasgow (Scotland): University of Strathclyde; 2002.
13. Eriksson U, Lemperg R. Roentgenological study of movements of the amputation stump within the prosthetic socket in below-knee amputees fitted with a PTB prosthesis. Acta Orthop Scand. 1969;40(4):520-29. [PMID: 5372254$]$

14. Grevsten S, Eriksson U. Stump-socket contact and skeletal displacement in a suction patellar-tendon bearing prosthesis. J Bone Joint Surg Am. 1974;56(8):1692-96. [PMID: 4434038]

15. Lilja M. Prosthetic fitting: Stump-socket interaction in transtibial amputees [dissertation]. Linkoeping (Sweden): Linkoeping University; 1998.

16. Newton RL, Morgan D, Schreiber MH. Radiological evaluation of prosthetic fit in below-the-knee amputees. Skeletal Radiol. 1988;17(4):276-80. [PMID: 3212490]

Submitted for publication August 30, 2005. Accepted in revised form June 23, 2006. 